\title{
ABOUT THE EXACT SOLUTION IN TWO PHASE-STEFAN PROBLEM
}

\author{
A. C. Boucíguez ${ }^{a}$, \\ R. F. Lozano ${ }^{a}$, \\ and M. A. Lara ${ }^{b}$ \\ ${ }^{\mathrm{a}}$ Facultad de Ciencias Exactas \\ Universidad Nacional de Salta, Argentina \\ Av. Bolivia 5150 - (4400) Salta - Argentina \\ Tel: $54-387-4255424$. \\ bouciga@unsa.edu.ar \\ lozanor@unsa.edu.ar \\ ${ }^{\mathrm{b}}$ Facultad de Ciencias Agrarias \\ Universidad Nacional de Rosario \\ Investigador del CONICET \\ IFIR. Instituto CONICET - UNR. \\ Pellegrini 250 - (2000) Rosario - Argentina. \\ malara@fceia.unr.edu.ar
}

ABSTRACT

Two cases of the two - phase Stefan problem in a semi - infinite slab are presented here: one has heat flux boundary condition proportional to $t^{-1 / 2}$ and the other has constant temperature boundary condition. In these two cases the exact solution exists, the relationship between the two boundary conditions is presented here, and the equivalence between the two problems is shown.

Keywords: two phase Stefan Problem, exact solution, heat flux boundary condition, temperature boundary condition.

\section{NOMENCLATURE}

c $\quad$ specific heat, $\mathrm{J} / \mathrm{kg}{ }^{\circ} \mathrm{C}$

$\mathrm{k}$ thermal conductivity, $\mathrm{W} / \mathrm{m}{ }^{\circ} \mathrm{C}$

$\mathrm{L} \quad$ latent heat of fusion, $\mathrm{J} / \mathrm{kg}$

$q(t) \quad$ heat flux, $\mathrm{W} / \mathrm{m}^{2}$

$s(t) \quad$ interface position, $\mathrm{m}$

$t \quad$ time independent variable, $\mathrm{s}$

$T(x, t)$ temperature profile of the phase change material, ${ }^{\circ} \mathrm{C}$

$T_{f} \quad$ melting temperature, ${ }^{\circ} \mathrm{C}$

$T_{0} \quad$ initial temperature, ${ }^{\circ} \mathrm{C}$

$u(x, t) \quad$ difference between $T(x, t)$ and $T_{f},{ }^{\circ} \mathrm{C}$

$u_{0} \quad$ difference between $T_{0}$ and $T_{f},{ }^{\circ} \mathrm{C}$

$x \quad$ spatial independent variable, $\mathrm{m}$

Ste Stefan number

\section{Greek symbols}

$\alpha \quad$ thermal diffusivity, $\mathrm{m}^{2} / \mathrm{s}$

$\rho$ density, $\mathrm{kg} / \mathrm{m}^{3}$

$\xi \quad$ similarity variable, $\mathrm{m} / \mathrm{s}^{1 / 2}$

$\lambda$ parameter to be determined, dimensionless

\section{Subscripts or superscripts}

$\begin{array}{ll}L & \text { liquid phase } \\ S & \text { solid phase } \\ f & \text { melting point } \\ 0 & \text { initial state }\end{array}$

0 initial state

\section{INTRODUCTION}

The free boundary problem presents many applications in physics and engineering. In particular, the so called "One and Two Phase Stefan Problem.”(Alexiades and Solomon, 1993) in a semi infinite slab is of great interest.

The one - phase Stefan Problem takes place when the initial phase is solid (liquid) at the melting point, the material is heated (cooled) from the side and, as a consequence, the temperature increases (decreases). The substance change to liquid (solid) phase in the vicinity of the boundary, leaving the rest of the solid (liquid) at the same initial temperature. This case was studied in a previous work (Boucíguez et al, 2006)

The two - phase Stefan Problem takes place when the initial phase is solid (liquid) at a temperature less (greater) than the melting one, the material is heated (cooled) from the side and as a consequence the temperature increases (decreases) to reach the melting point and then the liquid (solid) appears. In this case, the two phases: liquid and solid present a temperature distribution. These two temperature distributions and the interface position (free boundary) are unknown.

These two cases have exact solution when the condition in the boundary is: a constant temperature or the heat flux proportional to $t^{-1 / 2}$.

In a previous work (Boucíguez et al, 2006) it was shown that in the one - phase Stefan problem, the two boundary conditions are equivalent. Now, the equivalence between them will be shown for the two - phase Stefan problem. 
Figure 1 shows a scheme of the phase change material when solid is the initial condition, (a) at time $t=0$, and (b) at time $t>0$. If the initial condition is liquid the situation is equivalent, the words solid and liquid and the inequalities must be replaced in the figure.

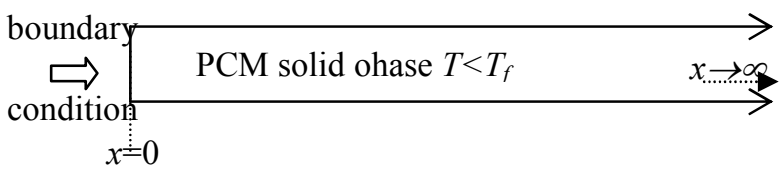

(a) phase change material at $t=0$

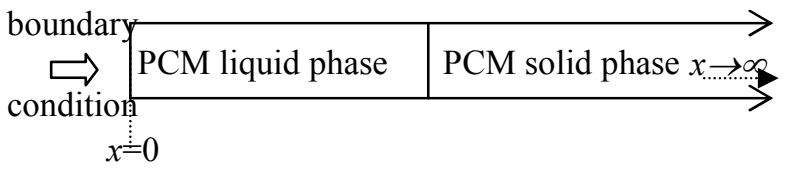

(b) phase change material at $t>0$

Figure 1. Scheme of the phase change material.

In a previous work (Lozano et al, 2003) it was observed that when the flux is $q(t)=q_{0} / t^{1 / 2}$, the temperature in the fixed face stays constant. In fact, in figures 2 to 5 , the temperature distribution for four different times $(5,10,15$, and 20 hours) are shown when $q_{0}$ is equal to $100,500,1000$, and 2000 $\mathrm{Ws}^{1 / 2} / \mathrm{m}^{2}$, respectively. The corresponding temperatures in the fixed face $(x=0)$ are constant and equal to $0.0001,0.0024,0.0098$, and $0.0392{ }^{\circ} \mathrm{C}$ respectively. These figures suggest a connection between the two boundary conditions and their equivalence has been shown here; that is to say: one is a consequence of the other and reciprocally

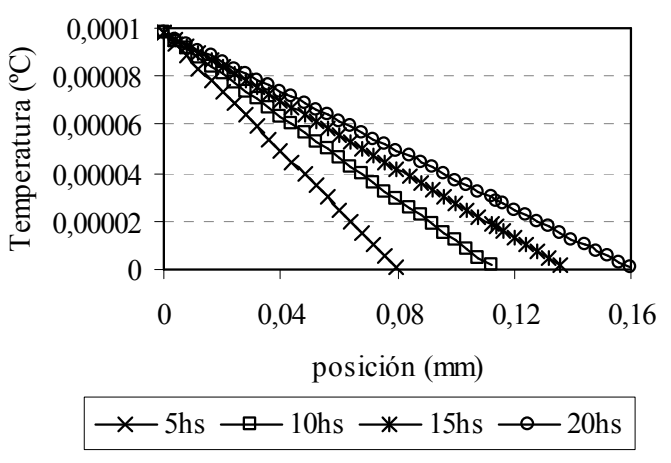

Figure 2. Temperature distribution for $q_{0}=100$ $\mathrm{Ws}^{1 / 2} / \mathrm{m}^{2}$.

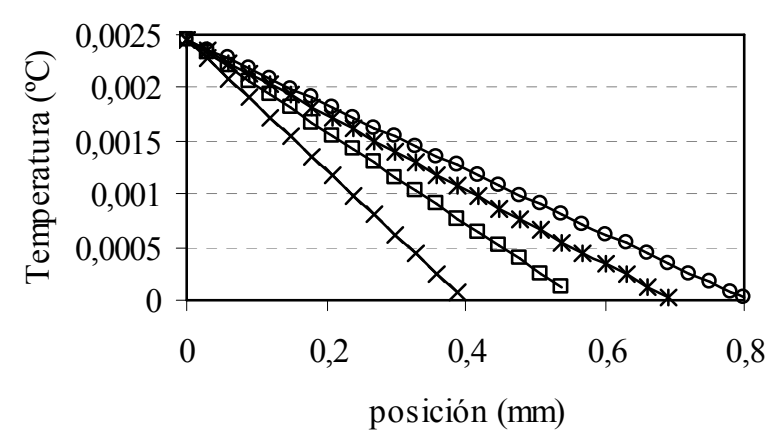

$\leftarrow 5 \mathrm{hs} \rightarrow-10 \mathrm{hs} \rightarrow-15 \mathrm{hs} \multimap-20 \mathrm{hs}$

Figure 3. Temperature distribution for $q_{0}=500$ $\mathrm{Ws}^{1 / 2} / \mathrm{m}^{2}$.

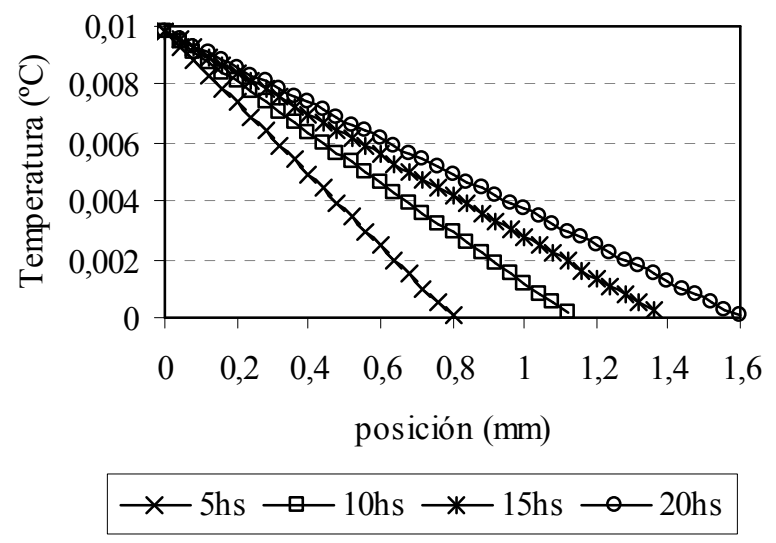

Figure 4. Temperature distribution for $q_{0}=1000$ $\mathrm{Ws}^{1 / 2} / \mathrm{m}^{2}$.

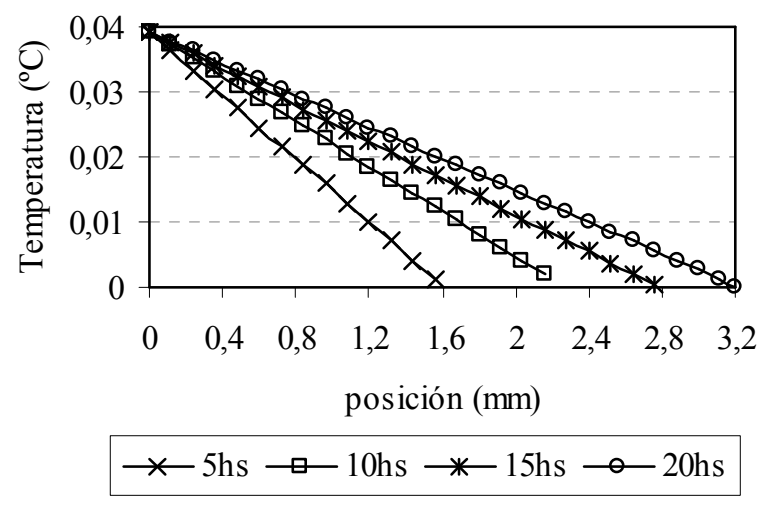

Figure 5. Temperature distribution for $q_{0}=2000$ $\mathrm{Ws}^{1 / 2} / \mathrm{m}^{2}$.

\section{MATHEMATICAL FORMULATION}

The two phase Stefan problem can be formulated as a heat conduction problem in a semi infinite slab, with two kind of boundary condition: constant temperature or heat flux proportional to $t^{-1 / 2}$. 
The problem is completely described by the following equation:

$$
\begin{aligned}
& \frac{\partial u^{L}}{\partial t}=\alpha_{L} \frac{\partial^{2} u^{L}}{\partial x^{2}}, \quad 0<t<\tau, \quad 0<x<s(t) \\
& \frac{\partial u^{S}}{\partial t}=\alpha_{S} \frac{\partial^{2} u^{S}}{\partial x^{2}}, \quad 0<t<\tau, \quad x>s(t) \\
& u^{L}(s(t), t)=u^{S}(s(t), t)=0, \quad \forall t>0, \quad 0<t<\tau \\
& \rho L \frac{d s(t)}{d t}=-k_{L} \frac{\partial u^{L}\left(s(t)^{-}, t\right)}{\partial x}+k_{S} \frac{\partial u^{S}\left(s(t)^{+}, t\right)}{\partial x} \\
& u^{S}(x, 0)=u_{S}<0, \quad 0<x \leq \infty>0, \quad 0<t<\tau \\
& s(0)=0
\end{aligned}
$$

and for the constant temperature case

$u^{L}(0, t)=u_{L}>0, \quad \forall t>0, \quad 0<t<\tau$

or for the heat flux proportional to $t^{-1 / 2}$

$$
k_{L} \frac{\partial u^{L}(0, t)}{\partial x}=-\frac{q_{0}}{\sqrt{t}}, \quad \forall t>0, \quad 0<t<\tau, q_{0}>0
$$

Where $\alpha$ is the thermal diffusivity, $k$ is the thermal conductivity, $\rho$ is the density, $c$ is the specific heat and $L$ is the latent heat of fusion. The index $L$ and $S$ (upper or sub) denote liquid and solid phase respectively.

The function $s(t)$, unknown a priori, is the interface position as a function of $t$; and $u(x, t)=T(x, t)$ $T_{f}$, is the difference between the substance temperature $T(x, t)$ and the fusion temperature $T_{f}$. At the same way $u_{L}=T_{L}-T_{f}$, is the difference between the temperature at the fixed face $T_{L}$, and $T_{f}$.

The Eq. 3 is the Stefan's equation, it represent the energy conservation on the interface position.

The Eqs. (6.1) and (6.2), show the two possible boundary conditions: constant temperature (6.1) and heat flux $\sim 1 / t^{1 / 2}(6.2)$. They are the only two cases where this problem has exact solution.

In the two cases, the analytical solution is obtained introducing the similarity variable $\xi$, (Alexiades and Solomon, 1993), defined by:

$\xi=\frac{x}{\sqrt{t}}$

The exact solution of the problem is obtained replacing (7) into Eqs. (1) to (6), hence the interface position results: $s(t)=2 \lambda \sqrt{\alpha_{L} t}$

Where $\lambda$ is a parameter to be determined for each case. For the constant temperature boundary condition, $\lambda$ is given by the equation:

$$
\lambda \cdot \sqrt{\pi}=\frac{S t e^{L}}{\exp \left(\lambda^{2}\right) \cdot \operatorname{erf}(\lambda)}-\frac{S t e^{S}}{v \exp \left(v^{2} \lambda^{2}\right) \cdot \operatorname{erf}(v \lambda)}
$$

and for the flux boundary condition, $\lambda$ is given by the equation:

$$
\lambda \cdot \sqrt{\pi}=\frac{q_{0}}{\rho \cdot L} \sqrt{\frac{\pi}{\alpha_{L}}} \frac{1}{\exp \left(\lambda^{2}\right)}-\frac{S t e^{S}}{v \exp \left(v^{2} \lambda^{2}\right) \cdot \operatorname{erf}(v \lambda)}
$$

where $\quad S t e^{L}=\frac{c_{L}}{L} u^{L}, \quad S t e^{S}=\frac{c_{S}}{L} u^{S}$ are the Stefan's number (liquid and solid, respectively) and $v=\sqrt{\frac{\alpha_{L}}{\alpha_{S}}}$.

The second term of the right side of Eqs. (9.1) and (9.2) are the same. They correspond to the solid phase, that is to say the initial one.

The two Eqs. (9.1) and (9.2) are transcendental. The solution of each one allows obtaining the exact solution of each problem. The solution for each equation is unique and it implies the uniqueness of the similarity solution. In consequence, the Stefan Problem admits only one solution.

The temperature distribution is given by the following equations: (Alexiades and Solomon, 1993) For the constant temperature boundary condition

$$
\begin{aligned}
& u^{L}(x, t)=u_{L}-u_{L} \cdot\left(\frac{\operatorname{erf}\left(\frac{x}{2 \sqrt{\alpha_{L} t}}\right)}{\operatorname{erf}(\lambda)}\right) \\
& u^{S}(x, t)=u_{S}-u_{S} \cdot\left(\frac{\operatorname{erfc}\left(\frac{x}{2 \sqrt{\alpha_{S} t}}\right)}{\operatorname{erfc}(v \lambda)}\right)
\end{aligned}
$$
$t^{-1 / 2}$

For the flux boundary condition proportional to 


$$
\begin{aligned}
& u^{L}(x, t)=\frac{q_{0}}{k_{L}} \cdot \sqrt{\pi \cdot \alpha_{L}} \cdot\left[\operatorname{erf}(\lambda)-\operatorname{erf}\left(\frac{x}{2 \sqrt{\alpha_{L} t}}\right)\right] \\
& u^{S}(x, t)=u_{S}-u_{S} \cdot\left(\frac{\operatorname{erfc}\left(\frac{x}{2 \sqrt{\alpha_{S} t}}\right)}{\operatorname{erfc}(v \lambda)}\right)
\end{aligned}
$$

The Eqs. (10.1.a) and (10.2.a) are equal to the one obtained for the one phase solution (Boucíguez et al, 2006). The Eq. (10.2.b) is equal to (10.1.b), that is to say the solid phase has the same expression for the two boundary conditions.

In all these equations $\operatorname{erf}(\xi)$ denotes the error function and $\operatorname{erfc}(\xi)$ denotes the complementary error function,

$$
\begin{aligned}
& f(\xi)=\operatorname{erf}(\xi)=\frac{2}{\sqrt{\pi}} \int_{0}^{\xi} \exp \left(-\theta^{2}\right) \cdot d \theta \\
& \operatorname{erfc}(\xi)=1-\operatorname{erf}(\xi)=\frac{2}{\sqrt{\pi}} \int_{x}^{\xi} \exp \left(-\theta^{2}\right) \cdot d \theta
\end{aligned}
$$

The basic properties of these functions are:

$$
\begin{aligned}
& f(0)=0 ; \quad f(\infty)=1 \\
& \frac{d f}{d \xi}=f^{\prime}(\xi)=\frac{2}{\sqrt{\pi}} \exp \left(-\xi^{2}\right)>0 \\
& \frac{d^{2} f}{d \xi^{2}}=f^{\prime \prime}(\xi)=-2 \cdot \xi \cdot f^{\prime}(\xi)=-\frac{4 \xi}{\sqrt{\pi}} \exp \left(-\xi^{2}\right) \\
& \operatorname{erfc}(0)=1 ; \quad \operatorname{erfc}(\infty)=0
\end{aligned}
$$

\section{RELATIONSHIP BETWEEN THE TWO} BOUNDARY CONDITIONS.

It is useful to name the parameter $\lambda$ in Eqs. (9) and (10), as $\lambda_{1}$ for the temperature condition and $\lambda_{2}$, for the flux condition; so the Eqs. (9) and (10) are written as:

For the temperature boundary condition:

$$
\lambda_{1} \cdot \sqrt{\pi}=\frac{S t e^{L}}{\exp \left(\lambda_{1}^{2}\right) \cdot \operatorname{erf}\left(\lambda_{1}\right)}-\frac{S t e^{S}}{v \exp \left(v^{2} \lambda_{1}^{2}\right) \cdot \operatorname{erf}\left(v \lambda_{1}\right)}
$$

For the flux boundary condition

$$
\lambda_{2} \cdot \sqrt{\pi}=\frac{q_{0}}{\rho \cdot L} \sqrt{\frac{\pi}{\alpha_{L}}} \frac{1}{\exp \left(\lambda_{2}^{2}\right)}-\frac{S t e^{S}}{v \exp \left(v^{2} \lambda_{2}^{2}\right) \cdot \operatorname{erf}\left(v \lambda_{2}\right)}
$$

as a consequence, Eqs. (10.1) and (10.2) become:

For the temperature boundary condition

$$
\begin{aligned}
& u^{L}(x, t)=u_{L}-u_{L} \cdot\left(\frac{\operatorname{erf}\left(\frac{x}{2 \sqrt{\alpha_{L} t}}\right)}{\operatorname{erf}\left(\lambda_{1}\right)}\right) \\
& u^{S}(x, t)=u_{S}-u_{S} \cdot\left(\frac{\operatorname{erfc}\left(\frac{x}{2 \sqrt{\alpha_{S} t}}\right)}{\operatorname{erfc}\left(v \lambda_{1}\right)}\right)
\end{aligned}
$$

For the flux boundary condition

$$
u^{L}(x, t)=\frac{q_{0}}{k_{L}} \cdot \sqrt{\pi \cdot \alpha_{L}} \cdot\left[\operatorname{erf}\left(\lambda_{2}\right)-\operatorname{erf}\left(\frac{x}{2 \sqrt{\alpha_{L} t}}\right)\right]
$$

$$
u^{S}(x, t)=u_{S}-u_{S} \cdot\left(\frac{\operatorname{erfc}\left(\frac{x}{2 \sqrt{\alpha_{S} t}}\right)}{\operatorname{erfc}\left(v \lambda_{2}\right)}\right)
$$

The equivalence between the two cases will be proved. Evaluating $u^{L}(0, t)$ from Eq. (10.2.a), results:

$$
u^{L}(0, t)=\frac{q_{0}}{k_{L}} \sqrt{\pi \cdot \alpha_{L}} \cdot \operatorname{erf}\left(\lambda_{2}\right)
$$

Then $u^{L}(0, t)$ is constant when the flux is proportional at $t^{-1 / 2}$, calling $u_{L}$ to this constant:

$$
u_{L}=\frac{q_{0}}{k_{L}} \cdot \sqrt{\pi \alpha_{L}} \cdot \operatorname{erf}\left(\lambda_{2}\right)
$$

Taking the derivative of the Eq. (10.1.a) with respect to $x$, and in virtue of the properties of error function, it yields

$$
u_{x}^{L}(x, t)=-u_{L} \cdot \frac{1}{\sqrt{\pi \alpha_{L}}} \cdot \frac{1}{\sqrt{t}} \cdot \exp \left(\frac{-x^{2}}{4 \alpha_{L} t}\right) \cdot \frac{1}{\operatorname{erf}\left(\lambda_{1}\right)}
$$


The heat flux at the boundary is $q(t)=-k_{L} u_{x}(0, t)$, hence evaluating the Eq. (15) at $x=0$, the heat flux results:

$$
q(t)=-k_{L} u_{x}(0, t)=k_{L} u_{L} \frac{1}{\sqrt{\pi \alpha_{L}}} \frac{1}{\operatorname{erf}\left(\lambda_{1}\right)} \frac{1}{\sqrt{t}}=\frac{c t e}{\sqrt{t}}
$$

calling $q_{0}$ to this constant, results:

$$
q_{0}=\frac{k_{L} \cdot u_{L}}{\sqrt{\pi \alpha_{L}}} \cdot \frac{1}{\operatorname{erf}\left(\lambda_{1}\right)}
$$

and as a consequence:

$$
u_{L}=\frac{q_{0}}{k_{L}} \cdot \sqrt{\pi \alpha_{L}} \cdot \operatorname{erf}\left(\lambda_{1}\right)
$$

replacing this value of $u_{L}$ at Eq. (10.1.a) results:

$$
u^{L}(x, t)=\frac{q_{0}}{k_{L}} \sqrt{\pi \alpha_{L}}\left[\operatorname{erf}\left(\lambda_{1}\right)-\operatorname{erf}\left(\frac{x}{2 \sqrt{\alpha_{L} t}}\right)\right]
$$

This last expression is similar to (10.2.a). The comparison between Eqs. (14) and (18), shows that, on one hand, they are formally the same and they are equal if and only if $\lambda_{1}=\lambda_{2}=\lambda$.

On the other hand, replacing $q_{0}$ from Eq. (17) in Eq. (10.2.a) it is obtained:

$$
u_{x}^{L}(x, t)=-u_{L} \cdot \frac{1}{\operatorname{erf}\left(\lambda_{1}\right)} \cdot\left[\operatorname{erf}\left(\lambda_{2}\right)-\operatorname{erf}\left(\frac{x}{2 \sqrt{\alpha_{L} t}}\right)\right]
$$

This two Eqs. (17) and (10.2.a) are equal if and only if $\lambda_{1}=\lambda_{2}=\lambda$.

Thus, it is shown that the two boundary conditions are equivalent.

\section{NUMERICAL EVALUATION}

A simple numerical evaluation of the situations discussed in the previous section is presented here, for different values $q_{0}$. The results are presented in Table 1; the second column is the value of $\lambda$ corresponding to Eq. (9.2), that is to say $\lambda_{2}$. The third column is the temperature obtained using this value and Eq. (10.2a). The fourth column is the value of $\lambda$ corresponding to Eq. (9.1), that is to say $\lambda_{1}$, evaluated with the value of $u(0, t)$ given in the third column.
Table 1 . Values of $\lambda_{1}, \lambda_{2}$, and boundary temperatures for different values of $q_{0}$.

\begin{tabular}{|c|c|c|c|}
\hline$q_{0}$ & $\lambda_{2}$ & $u(0, t)$ & $\lambda_{1}$ \\
\hline 100 & 0,00078215 & 0,0000981 & $7,821510^{-4}$ \\
\hline 500 & 0,0039107 & 0,002152 & $3,910710^{-3}$ \\
\hline 1000 & 0,0078210 & 0,009809 & $7,82110^{-3}$ \\
\hline 2000 & 0,0156391 & 0,039228 & $1,563910^{-2}$ \\
\hline 3000 & 0,0234515 & 0,088228 & $2,345210^{-2}$ \\
\hline 4000 & 0,0312554 & 0,156761 & $3,125510^{-2}$ \\
\hline 5000 & 0,0390478 & 0.244761 & $3,904810^{-2}$ \\
\hline
\end{tabular}

In the same way, Table 2 shows, the numerical results for different temperature boundary conditions and the initial one. The first column is the temperature boundary condition, the second the initial one, the third is the $\lambda$ value obtained of Eq. (9.1), that is to say $\lambda_{1}$. The fourth column is the obtained value of $q_{0}\left(\mathrm{~W} \mathrm{~s}^{1 / 2} / \mathrm{m}^{2}\right)$ using this $\lambda$ value. The fifth column is the $\lambda$ value evaluated using the obtained value of $q_{0}$ and the initial temperature using Eq. (9.2), that is to say $\lambda_{2}$.

Table 2. Values of $\lambda_{1}, \lambda_{2}, q_{0}$ for different values of temperatures boundary and initial conditions.

\begin{tabular}{|c|c|c|c|c|}
\hline$u_{L}$ & $\mathrm{u}_{0}$ & $\lambda_{1}$ & $q_{0}$ & $\lambda_{2}$ \\
\hline 20 & -2 & 0.32659 & $1.262610^{4}$ & 0.32659 \\
\hline 20 & -5 & 0.35419 & $1.171310^{4}$ & 0.35419 \\
\hline 25 & -2 & 0.35788 & $1.450210^{4}$ & 0.35788 \\
\hline 25 & -5 & 0.38259 & $1.364610^{4}$ & 0.38259 \\
\hline 30 & -2 & 0.38589 & $1.624810^{4}$ & 0.38589 \\
\hline 30 & -5 & 0.40834 & $1.544210^{4}$ & 0.40834 \\
\hline
\end{tabular}

All these calculations where made using the Mathematical Support of the Scientific Work Place, so the precision is ensured. The results showed in both tables allow to say that the two values $\lambda_{1}$ and $\lambda_{2}$, obtained by different ways are equal, as it was proved in the last section.

\section{DISCUSSION AND CONCLUSION}

The analysis of Figures 2 to 5, for a particular material (organic wax), shows that the heat flux $\mathrm{q}_{0} / t^{1 / 2}$ produces a constant temperature in the fixed face. The obtained results are summarized in Table 3. The first column is the value of $q_{0}$, the second one is the obtained result for $u(0, t)$ from Eq. (10.2.a), using this $q_{0}$, and the corresponding value of $\lambda_{2}$. The third column is the relationship between that $q_{0}$ and the minor value $\left(q_{0}=100\right)$, that is to say, it was obtained taking 100 , as the first value. Thus, for $q_{0}=500$, this factor is $500 / 100=5$, for $q_{0}=1000$, is $1000 / 100=10$ and so on. The fourth column is the ratio between $u(0, t)$ and the value of $u(0, t)$ corresponding to $q_{0}=100$. Finally the fifth column is the ratio between the fourth and second columns. 
Table $3 . q_{0}$ values and the corresponding boundary temperature.

\begin{tabular}{|c|c|c|c|c|}
\hline$q 0$ & $u(0, t) \mathrm{C}$ & & & \\
\hline 100 & 0.00010 & 1 & 1 & 1 \\
\hline 500 & 0.0025 & 5 & 25 & 5 \\
\hline 1000 & 0.010 & 10 & 100 & 10 \\
\hline 2000 & 0.04 & 20 & 400 & 20 \\
\hline
\end{tabular}

These results together with those show in Tab. 1 and 2, validate the equivalence between the two boundary conditions.

Table 3 also shows that when $q_{0}$ increases, the temperature in the fixed face is multiplied by the squared ratio between the new and the old $q_{0}$ (third column)

This ratio only depends on $q_{0}$, given that it is the only factor that changes in Eq. (10.2) for different materials. Thus, this relationship is also valid for all substances.

Thus, it is proved that the two well known analytical solutions for the two - phases Stefan Problem, one for temperature boundary condition, the other for heat flux condition are not independent: one determines the other.

\section{ACKNOWLEDGEMENTS}

The authors are grateful to Dra. Marta C. Pocoví for the English revision.

This paper has been financed by Investigation Project $\mathrm{N}^{\mathrm{o}}$ 1264, CIUNSa. National University of Salta, Argentina.

\section{REFERENCES}

Alexiades, V and Solomon, A. Mathematical Modeling of Melting and Freezing Processes. Hemisphere Publishing Corporation, 1993.

Boucíguez, A; Lozano, R and Lara, M. Some Considerations Regarding the Exact Solution in One Phase Stefan Problem (Engenharia Termica, in press) Lozano, R; Boucíguez, A; Lara, M and Villa, L. 2003. Comportamiento de la frontera Libre en Materiales de Cambio de Fase con Condiciones de Flujo de Calor Constante. Revista Energías Renovables y Medio Ambiente, Vol. 12, p. 49 - 53. 